\title{
Pharmacogenomics in psychiatry: clinical issues to be considered
}

\author{
Molecular Psychiatry (2005) 10, 615. \\ doi:10.1038/sj.mp.4001700
}

Pharmacogenomics is aimed at optimizing treatment and identifying new targets for drug development. However, for research in this field to advance and for its key promises to become a reality, it is necessary that we identify and address essential issues. Those are broadly defined into two groups. First, there are topics related to clinical research design and then we should consider genomic approaches. Expertise in these two areas is essential. We often see outstanding clinical protocols that are followed by a mediocre approach to genetics, and we see outstanding genetics conducted on poorly conceptualized clinical approaches. Neither type of study yields useful data.

Considerations that emerge as clinical studies are designed include choice of outcomes. Should those be categorical (responder vs nonresponder) or continual (such as a Hamilton scale)? If categorical, how are they defined? And based on what criteria? If one opts for continual outcomes, how can ratings be done consistently and reliably over time?

The correct assignment of phenotypes is crucial. One could include in a study a patient with family history of the disease, let us say depression, and a history of chronic depression with multiple episodes. According to most protocols you could also include in the same study a person without family history, in a new episode of 2-3 weeks' duration. These individuals meet the DSM-IV criteria for major depression, but do they have the same disease?

Once patients are recruited, how will they be treated? The best design is of course a standardized, double-blind, prospective, placebo-controlled study, with drugs that are well used and well established. This becomes difficult at several levels. Drug company protocols are aimed at studying new compounds. The optimal pharmacogenomic study would have a large impact by studying widely used drugs of direct public health relevant. The topic of sample size emerges here as crucial. Large and highly costly treatment groups are needed so that sufficient power exists for the application of existing technology. Pharmaceutical companies are unlikely to cover the costs of large studies of drugs that are already on the market, selling well, and close to or at the end of their patents. Federal funding agencies tend to shy away from expensive 'clinical trials' that from the geneticist's perspective are subsumed under the highly inappropriate concept of 'sample collection.' Genomic-based companies likewise are looking for low cost samples on which they apply their sophisticated new methods. According to popular wisdom, 'the cheap becomes expensive.' We would propose that the paucity of conclusive results is a direct consequence of the unwillingness of funding sources to spend what it really takes to generate optimally collected genetic material with rigorously characterized phenotypic measures of the underlying disease and of treatment responses. The eagerness to economize on the clinical, nonhypotheses-based, nontechnology-intensive side of the research has led to considerable wasted effort by highly talented geneticists and statisticians.

Treatment compliance is critical. The assumption is that the drug is taken and variability is then associated to genes. If the drug is not taken, or taken irregularly, the patterns of response are more easily attributable to compliance than to genomics. Think about the last time you were prescribed a course of medication. Did you, a highly educated professional, comply rigorously and consistently with treatment? When we are prescribed something as short term and medically necessary as antibiotics, we forget doses and times, in spite of our best efforts and full understanding of emerging drug resistance. How consistently are depression, schizophrenia, and other mental disorders treated, particularly as their symptoms directly impact on the ability to follow treatment consistently? Those symptoms affect volition, attention, concentration, motivation, affect, and cognition.

Placebo responses are also particularly problematic. Is it ethical to have a full placebo group in a study of established drugs, which have been extensively proved to be effective? Moreover, in the drug groups, there will be a mixture of placebo and drug responders. For pharmacogenomics, it is not sufficient to know the percentage of placebo responses. It would be essential to differentiate placebo from drug responders within the drug-treated group. However, that is not feasible at present.

Additional issues to be considered include environmental contributions to treatment outcome, excessive expectations, confidentiality, and community consultation and engagement.

A future editorial will comment on the genomic issues that have to be expertly addressed after the complex clinical topics discussed here are fully considered. 